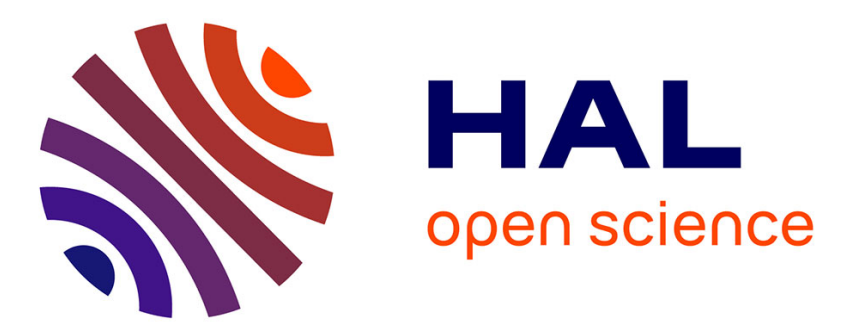

\title{
Mesoscopic constitutive law with nonlinear elasticity and phase transformation for the twinning-buckling of TATB under dynamic loading
}

\author{
Paul Lafourcade, Christophe Denoual, Jean-Bernard Maillet
}

\section{To cite this version:}

Paul Lafourcade, Christophe Denoual, Jean-Bernard Maillet. Mesoscopic constitutive law with nonlinear elasticity and phase transformation for the twinning-buckling of TATB under dynamic loading. Physical Review Materials, 2019, 3 (5), 10.1103/PhysRevMaterials.3.053610 hal-03417481

\author{
HAL Id: hal-03417481 \\ https://hal.science/hal-03417481
}

Submitted on 29 Nov 2021

HAL is a multi-disciplinary open access archive for the deposit and dissemination of scientific research documents, whether they are published or not. The documents may come from teaching and research institutions in France or abroad, or from public or private research centers.
L'archive ouverte pluridisciplinaire $\mathbf{H A L}$, est destinée au dépôt et à la diffusion de documents scientifiques de niveau recherche, publiés ou non, émanant des établissements d'enseignement et de recherche français ou étrangers, des laboratoires publics ou privés. 


\title{
Mesoscopic constitutive law with nonlinear elasticity and phase transformation for the twinning-buckling of TATB under dynamic loading
}

\author{
Paul Lafourcade, ${ }^{*}$ Christophe Denoual, and Jean-Bernard Maillet \\ CEA-DAM, DIF, F-91297 Arpajon, France
}

(Received 8 April 2019; published 31 May 2019)

\begin{abstract}
A mesoscopic constitutive law for 1,3,5-triamino-2,4,6-trinitrobenzene (TATB), an ultra-anisotropic energetic molecular crystal, is proposed and validated on molecular dynamics (MD) simulations. The two dominant deformation mechanisms observed at nanometric scale and limited stress (less than $1 \mathrm{GPa}$ ) are a buckling instability and a nonsymmorphic twinning (irreversible) transformation. A thermodynamically consistent continuum model is detailed, with nonlinear elasticity in pressure constructed to reproduce a cold equation of state. The twinning-buckling phase transition observed in MD is modeled by using a phase field by reaction pathway (PF-RP) formalism. In order to validate the present constitutive law, we first study the response of the single crystal under constant-strain-rate uniaxial compressions for various directions in the basal plane and present one-to-one comparisons between MD and PF-RP simulations. As an upscale case for the constitutive law, a large polycrystal is subjected to a shock compression at low velocity and the activation of the twinning-buckling mechanism is discussed.
\end{abstract}

DOI: 10.1103/PhysRevMaterials.3.053610

\section{INTRODUCTION}

Ultra-anisotropic materials, characterized by differences of the directional Young modulus by at least one order of magnitude, possess an unusual mechanism of deformation, an elastic instability called buckling. Although purely of elastic nature, this instability remains challenging to model, mainly due to the need of strongly nonlinear elastic behavior for the compression in the hard directions as well as large strain formalism to accurately represent the elastic rotations induced by the buckling. This instability generates large deformations localized in thin strips, which, in the case of molecular crystals, could modify the local molecular order and thus induce a phase change or a twinning. The overall behavior could therefore be a complex compound of nonlinear elasticity and twinning, both in large-strain formalism.

Such coupling has been found to be the predominant mechanism at small scale for 1,3,5-triamino-2,4,6-trinitrobenzene (TATB), an ultra-anisotropic molecular crystal [1]. The TATB crystal is centrosymmetric and crystallizes in a triclinic unit cell of space group $P \overline{1}[2,3]$ containing two molecules, with a hexagonal-like stacking of planar molecules (see Fig. 1). This layered structure, as well as the important contrast between interplanar interactions (governed by van der Waals interactions) and the strong hydrogen-bond network present in the molecular layers, confers some ultra-anisotropic properties [4,5] to this crystal. Indeed, various computational studies of the second-order stiffness tensor $\boldsymbol{C}$ [1,6-8] have highlighted a ratio of 12 for the elastic shear components. Despite its great importance in the field of energetics materials, very few experiments at the single-crystal scale have been performed, mainly due to its poor solubility making it difficult to obtain

*paul.lafourcade@cea.fr high-quality single crystals, as well as for security constraints, fostering the need of an accurate modeling.

At a more macroscopic scale, experimental observations have shown that TATB grown single crystals contain many defects such as porosity, wormhole networks, and twinned structures [9-12] that may have consequences on their mechanical behavior and their sensitivity, i.e., the ability of energetic materials to remain stable during shock loading. This sensitivity depends on complex mechanisms such as deformation localization [13] involved in the process of hot spots [14-17]. The latter are known to be activated with local plastic activity, dislocation pileup [18], void collapse $[19,20]$, localized heating due to the nucleation of defects [15], or friction of cracks (present in polycrystalline TATB, as suggested by [21], and induced by its strong anisotropy). A very detailed review of shock and detonation properties of high explosives has been recently proposed by Handley et al. [22], in which the authors discuss the building of mesoscale models and their application to explosive phenomena.

A few experimental studies have been conducted to characterize the behavior of a TATB-based plastic bonded explosive $(\mathrm{PBX})$ under pressure through the measurements of its equation of state (EOS): Olinger and Cady [23] obtained X-ray powder diffraction (XRD) patterns up to $7 \mathrm{GPa}$ using a loosely inserted powder in the diamond anvil cell (DAC) leading to hydrostatic pressure conditions. Stevens et al. [24] performed XRD experiments using a DAC on grounded TATB crystals immersed in a methanol/ethanol leading to similar pressure conditions. In a recent study, Plisson et al. [3] determined the EOS of TATB up to $66 \mathrm{GPa}$ using synchrotron XRD experiments with a DAC using a few TATB single crystals surrounded by neon as a pressure-transmitting medium. These three experiments reflect that the hydrostatic pressure conditions were mainly respected even if different samples were 

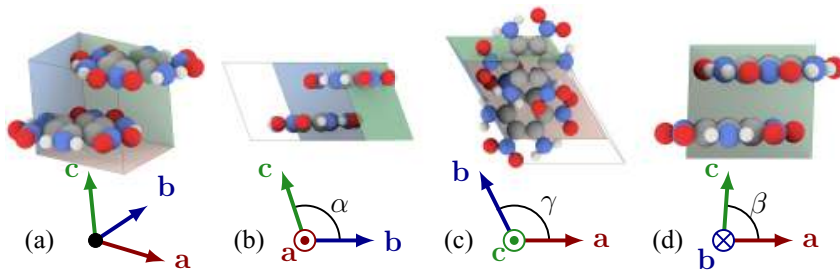

FIG. 1. Crystal structure. TATB unit cell with lattice vectors $\boldsymbol{a}, \boldsymbol{b}, \boldsymbol{c}$ and cell angles $\alpha, \beta$, and $\gamma$. (a) 3D representation. (b) $x+$ view. (c) $z+$ view. (d) $y+$ view. Atom colors are white for hydrogen, gray for carbon, blue for nitrogen, and red for oxygen.

used (i.e., powder vs multiple single crystals). An interesting result is that TATB did not undergo any phase transformation during these loadings and remained stable while conserving its high anisotropy. A few computational studies involving ab initio calculations $[3,8]$ and classical molecular dynamics (MD) simulations [1,6] have been performed and proved to be in good agreement with the experiments.

MD simulations of the TATB single crystal have been widely used recently and play a crucial role in predicting its mesoscopic behavior. The thermal properties and idealized hot spot relaxation have been studied by Kroonblawd et al. $[4,5,14]$, while Mathew et al. calculated the generalized stacking fault energies of TATB basal planes [7], their elasticplastic response under displacement-controlled nanoindentation [18], and more recently their liquid-transport properties [25]. The results on the response under nanoindentation are found to be in good agreement with the recent experiments of Taw et al. [26], which is the only TATB single crystal mechanical experiment performed up to date. Long and Chen also studied interfacial properties for PBX and explosive/additive materials $[27,28]$ as well as hot-spot formation in energetic materials [29]. Dislocations are known to be responsible for plasticity in crystalline materials; such defects have been studied by Lafourcade et al. [1], who presented results on dislocation core structures in the TATB basal plane in temperature and pressure. They concluded that dislocations split into three or more partials, leading to large stacking fault ribbons of approximately 50-100 unit cells. More recently, Lafourcade et al. [30] computed a directional nucleation stress surface under pure shear for the TATB single crystal through MD simulations, leading to the identification of three distinct behaviors: (i) Basal shear is very easy to activate due to the low stacking fault energies in the basal plane, (ii) nucleation of nonbasal dislocations occurs at a yield stress around $1.5 \mathrm{GPa}$ leading to a very complex core structure with a local dilatancy, and (iii) an instability (termed in the following the "buckling instability") is shown to occur for moderate compression (a few percent) in the basal plane. This instability can be accompanied by a twinning irreversible deformation for some directions of compression. The latter deformation mechanism can be activated at a longitudinal strain of $\simeq 5 \%$ and at a nucleation stress much lower than the one required for nonbasal dislocation nucleation. Furthermore, they found that a perfect twinning mechanism could be obtained by applying pure shear to the basal plane with a maximum von Mises shear stress around $150 \mathrm{MPa}$ along the transformation pathway.
In this paper, we address the lack of continuum modeling of the TATB single crystal by presenting a constitutive law that includes two important features: a nonlinear elasticity to model the high compliance of the soft directions, and a large-strain formalism to allow for large rotations involved in the resulting wavelike structure. Moreover, the nonlinear elasticity formalism presented in this paper is built to reproduce the cold equation of state (EOS) obtained through MD simulations. A coupling is proposed with a phase field by reaction pathway (PF-RP) formalism to model the twinning transition, described in [30] as a consequence of the buckling, as a dissipative process. This PF-RP formalism was first introduced by Denoual [31] to model martensitic transformations and subsequently applied to the polymorphisms or iron at high pressure $[32,33]$. Finally, two assumptions were made prior to the construction of the present constitutive law. First, dislocations in the basal plane were not considered in the constitutive law as they result in large stacking fault ribbons that exceed the resolution of the following continuum model. Second, nonbasal dislocations were not taken into account since they require a nucleation stress much higher than the stress needed to activate the twinning-buckling mechanism, which is thought to be predominant in a first approximation [30]. In addition, during all the MD simulations presented in this work, nucleation of dislocations was never observed, therefore justifying the exclusion of crystal plasticity in our model, which aims to reproduce the presented MD simulations.

The theory used in this work is based on a coupling between the Landau free energy description [34] and elastic deformations via a thermodynamically consistent finite-strain formalism. Inelastic energies (see, e.g., [32]) of the reaction pathway that links TATB phases (stable and twinned) are inferred by MD simulations as well as the pressuredependent elastic properties. Continuum mechanics equations of motion as well as transformational strains based on time-dependent Ginzburg-Landau equations [35] are derived in a Lagrangian formulation and the model is implemented into an element-free Galerkin (EFG) Lagrangian code.

The paper is organized as follows. The first section is dedicated to the nonlinear elasticity formalism to define a pressure-dependent second-order stiffness tensor from an equation of state computed through MD simulations. The second section aims at presenting the PF-RP model applied to the shear-induced twinning transformation, inferred by MD results from Lafourcade et al. [30]. One-to-one comparisons between classical MD and continuum mechanics simulations are presented in the third section. Both uniaxial and spherical (i.e., triaxial) compressions on a single crystal are simulated in order to validate the constitutive law, leading to a complex microstructure that couples simple buckling and twinning transitions. Finally, the last section is dedicated to the use of the constitutive law to simulate a TATB polycrystal under shock loading, at a scale not reachable by classical molecular dynamics. As notations, we use "." for the matrix product, ":" for the matrix inner product (the inner product takes precedence over the simple product), $a^{\prime}$ for the derivative of $a, \dot{a}$ for the time derivative, and $\ddot{a}$ for the double time derivative. 


\section{EQUATION OF STATE AND NONLINEAR ELASTICITY}

\section{A. Kinematics}

Let $\boldsymbol{X}$ be a material point in a homogeneous reference configuration $\Omega_{0} \subset \mathbb{R}^{3}$. The evolution over time of $\Omega_{0}$ is governed by the mapping $\boldsymbol{x}=\chi(\boldsymbol{X}, t): \Omega_{0} \longrightarrow \Omega \subset \mathbb{R}^{3}$ with respect to time $t \in \mathbb{R}^{+}$. The current configuration is described by the total deformation gradient tensor $\boldsymbol{F}$, which is expressed using a multiplicative decomposition [33,36-39]:

$$
\boldsymbol{F}=\left.\frac{\partial \chi}{\partial \boldsymbol{X}}\right|_{t}=\nabla \chi=\boldsymbol{F}_{\mathrm{e}} \cdot \boldsymbol{F}_{\mathrm{t}},
$$

with $\nabla$ the material derivative with respect to $\boldsymbol{X}$. The reference configuration represents the initial defect-free TATB single crystal, and as the crystal undergoes deformation, the shearinduced twinning is described by the transformation deformation gradient tensor $\boldsymbol{F}_{\mathrm{t}}$. Finally, the current configuration is reached by elastic distortion and rigid-body rotation, both included in the elastic deformation gradient tensor $\boldsymbol{F}_{\mathrm{e}}$ through $\boldsymbol{U}_{\mathrm{e}}$ and $\boldsymbol{R}_{\mathrm{e}}$, respectively. This polar decomposition reads $\boldsymbol{F}_{\mathrm{e}}=$ $\boldsymbol{R}_{\mathrm{e}} \cdot \boldsymbol{U}_{\mathrm{e}}$, with $\boldsymbol{U}_{\mathrm{e}}^{2}=\boldsymbol{F}_{\mathrm{e}}^{T} \cdot \boldsymbol{F}_{\mathrm{e}}$ and $\operatorname{det} \boldsymbol{F}_{\mathrm{e}}=\operatorname{det} \boldsymbol{U}_{e}=j_{\mathrm{e}}$, where the superscript ${ }^{T}$ is used as the transposition operator. The volume change conditions used in the present work are the following:

$$
j_{\mathrm{e}}=\operatorname{det} \boldsymbol{F}_{\mathrm{e}}>0, \quad j_{\mathrm{t}}=\operatorname{det} \boldsymbol{F}_{\mathrm{t}}=1,
$$

leading to the invertibility of $\boldsymbol{F}_{\mathrm{e}}$ and $\boldsymbol{F}_{\mathrm{t}}$, the latter being a volume-preserving transformation. According to the conservation of mass, the determinant of $\boldsymbol{F}$ gives the volume change between current and reference configurations, with respective volumes $V$ and $V_{0}$, i.e., $\operatorname{det} \boldsymbol{F}=j=\operatorname{det} \boldsymbol{F}_{\mathrm{e}}=j_{\mathrm{e}}=\rho_{0} / \rho=$ $V / V_{0}$, where $\rho$ and $\rho_{0}$ are the current and reference mass density, respectively.

\section{B. Application of thermodynamics laws}

Equilibrium equations of total forces must be satisfied during the deformation evolution in a Lagrangian formulation; the balance of linear momentum is given by

$$
\boldsymbol{\nabla} \cdot \boldsymbol{P}+\rho_{0} \boldsymbol{b}=\rho_{0} \ddot{\boldsymbol{u}} \quad \text { in } \quad \Omega_{0},
$$

where $\boldsymbol{P}$ is the first Piola-Kirchhoff stress tensor, $\boldsymbol{b}$ is the external body force density, $\ddot{\boldsymbol{u}}=\ddot{\chi}(\boldsymbol{X}, t)$ is the material time acceleration of point $\boldsymbol{X}$ associated with the corresponding displacement field $\boldsymbol{u}=\chi(\boldsymbol{X}, t)-\boldsymbol{X}$, and $\boldsymbol{\nabla} \cdot$ is the divergence operator.

The coupling between large elastoplastic deformations and the phase-field approach derives from a thermodynamic framework governed by the second law of thermodynamics. While ignoring thermal effects, the Clausius-Duhem inequality can be expressed in terms of stress power per unit reference volume [40] as follows:

$$
\int_{\Omega_{0}}\left(\boldsymbol{P}: \dot{\boldsymbol{F}}-\rho_{0} \dot{\psi}\right) d \Omega_{0} \geqslant 0,
$$

where ":" is the double inner tensor product and $\psi$ translates the Helmholtz free energy per unit mass. From Eq. (4), it follows that the first Piola-Kirchhoff stress tensor $\boldsymbol{P}$ and the total deformation gradient tensor $\boldsymbol{F}$ are work-conjugate variables, with $\boldsymbol{P}: \dot{\boldsymbol{F}}$ being the mechanical stress power per unit reference volume. Using the multiplicative decomposition of Eq. (1), it is ideally proposed that the Helmholtz free energy reads as follows:

$$
\psi=\psi\left(\boldsymbol{F}_{\mathrm{e}}, \boldsymbol{F}_{\mathrm{t}}\right)=\psi_{\mathrm{e}}\left(\boldsymbol{F}_{\mathrm{e}}\right)+\psi_{\mathrm{t}}\left(\boldsymbol{F}_{\mathrm{t}}\right),
$$

where it is assumed that elastic and transformational distortions are uncoupled events, so that the free energy is additively decomposed into elastic $\psi_{\mathrm{e}}$ and transformational $\psi_{\mathrm{t}}$ contributions. From Eqs. (1) and (5), the rates of total deformation and free energy can be obtained, i.e.,

$$
\begin{aligned}
& \dot{\boldsymbol{F}}=\dot{\boldsymbol{F}}_{\mathrm{e}} \cdot \boldsymbol{F}_{\mathrm{t}}+\boldsymbol{F}_{\mathrm{e}} \cdot \dot{\boldsymbol{F}}_{\mathrm{t}}, \\
& \dot{\psi}=\frac{\partial \psi_{\mathrm{e}}}{\partial \boldsymbol{F}_{\mathrm{e}}}: \dot{\boldsymbol{F}}_{\mathrm{e}}+\frac{\partial \psi_{\mathrm{t}}}{\partial \boldsymbol{F}_{\mathrm{t}}}: \dot{\boldsymbol{F}}_{\mathrm{t}} .
\end{aligned}
$$

Inserting Eqs. (6) into the Clausius-Duhem inequality (4), the latter leads to

$$
\begin{aligned}
& \int_{\Omega_{0}}\left\{\left(\boldsymbol{P} \cdot \boldsymbol{F}_{\mathrm{t}}^{T}-\rho_{0} \frac{\partial \psi_{\mathrm{e}}}{\partial \boldsymbol{F}_{\mathrm{e}}}\right): \dot{\boldsymbol{F}}_{\mathrm{e}}\right. \\
& \left.+\left(\boldsymbol{F}_{\mathrm{e}}^{T} \cdot \boldsymbol{P}-\rho_{0} \frac{\partial \psi_{\mathrm{t}}}{\partial \boldsymbol{F}_{\mathrm{t}}}\right): \dot{\boldsymbol{F}}_{\mathrm{t}}\right\} d \Omega_{0} \geqslant 0 .
\end{aligned}
$$

A local formulation of the free-energy imbalance $\mathcal{D}$ can be written as

$$
\mathcal{D}=\left(\boldsymbol{P} \cdot \boldsymbol{F}_{\mathrm{t}}^{T}-\rho_{0} \frac{\partial \psi_{\mathrm{e}}}{\partial \boldsymbol{F}_{\mathrm{e}}}\right): \dot{\boldsymbol{F}}_{\mathrm{e}}+\boldsymbol{X}_{\mathrm{t}}: \dot{\boldsymbol{F}}_{\mathrm{t}} \geqslant 0,
$$

where the term $\boldsymbol{X}_{\mathrm{t}}$, conjugated to the dissipative rate $\dot{\boldsymbol{F}}_{\mathrm{t}}$, is the dissipative forces given by

$$
\boldsymbol{X}_{\mathrm{t}}=\boldsymbol{F}_{\mathrm{e}}^{T} \cdot \boldsymbol{P}-\rho_{0} \frac{\partial \psi_{\mathrm{t}}}{\partial \boldsymbol{F}_{\mathrm{t}}} .
$$

Thus, a change in $\boldsymbol{F}_{\mathrm{t}}$ leads to the thermodynamic displacive driving forces defined by Eq. (9), acting on a material point $\boldsymbol{X}$. A standard assumption consists in considering nondependency between the rate of dissipation and $\dot{\boldsymbol{F}}_{\mathrm{e}}$ in Eq. (8), i.e., the nondissipative property of elasticity. This results in the following expression of the first Piola-Kirchhoff stress tensor:

$$
\boldsymbol{P}=\rho_{0} \frac{\partial \psi_{\mathrm{e}}}{\partial \boldsymbol{F}_{\mathrm{e}}} \cdot \boldsymbol{F}_{\mathrm{t}}^{-T} .
$$

\section{Constitutive equations}

\section{Hyperelasticity}

The elastic part of the Helmholtz free energy is assumed to be a function of both the Green-Lagrange strain tensor $\boldsymbol{E}_{\mathrm{e}}$ and the volume-dependent stiffness tensor $\mathbb{D}(\operatorname{det} \boldsymbol{F})$, i.e.,

$$
\rho_{0} \psi_{\mathrm{e}}=\frac{1}{2} \boldsymbol{E}_{\mathrm{e}}: \mathbb{D}(\operatorname{det} \boldsymbol{F}): \boldsymbol{E}_{\mathrm{e}},
$$

where $\boldsymbol{E}_{\mathrm{e}}=\frac{1}{2}\left(\boldsymbol{C}_{\mathrm{e}}-\boldsymbol{I}\right)$ with $\boldsymbol{C}_{\mathrm{e}}=\boldsymbol{F}_{\mathrm{e}}^{T} \cdot \boldsymbol{F}_{\mathrm{e}}$ being the right Cauchy-Green strain tensor. Inserting Eq. (11) into Eq. (10) leads to the nonlinear stress-strain relation in terms of the first Piola-Kirchhoff stress tensor,

$$
\boldsymbol{P}=\boldsymbol{F}_{\mathrm{e}} \cdot \mathbb{D}: \boldsymbol{E}_{\mathrm{e}} \cdot \boldsymbol{F}_{\mathrm{t}}^{-T}+\frac{1}{2} \operatorname{det} \boldsymbol{F} \boldsymbol{E}_{\mathrm{e}}: \mathbb{D}^{\prime}: \boldsymbol{E}_{\mathrm{e}} \cdot \boldsymbol{F}^{-T},
$$

and Cauchy stress tensor,

$$
\sigma=j^{-1} \boldsymbol{P} \boldsymbol{F}^{T} .
$$




\section{Volume-dependent elasticity}

The EOS, which links volume variations and pressure, is used in this section to define the evolution of $\mathbb{D}(\operatorname{det} \boldsymbol{F})$, assumed to be proportional to the fourth-order elastic tensor at ambient temperature and pressure $\mathbb{C}_{0}$ :

$$
\mathbb{D}(\operatorname{det} \boldsymbol{F})=k(\operatorname{det} \boldsymbol{F}) \cdot \mathbb{C}_{0},
$$

where $\mathbb{C}_{0}$ is the stiffness tensor at pressure $p=0$ computed with MD simulations [1], and $k$ a scalar function to be determined.

Along a prescribed spherical deformation $\boldsymbol{F}=(\operatorname{det} \boldsymbol{F})^{1 / 3} \boldsymbol{I}$, the first Piola stress tensor $\boldsymbol{P}$ reads

$$
\boldsymbol{P}=\boldsymbol{F} \cdot \mathbb{D}: \boldsymbol{E}+\operatorname{det} \boldsymbol{F} \frac{e^{2}}{2} \boldsymbol{I}: \mathbb{D}^{\prime}: \boldsymbol{I F}^{-T}
$$

with $e=\frac{1}{2}\left[(\operatorname{det} \boldsymbol{F})^{2 / 3}-1\right]$ and $\boldsymbol{E}=e \boldsymbol{I}$. Then, by stating that the pressure $p=-\operatorname{trace}(\sigma) / 3$ follows the cold equation of state $P_{\mathrm{EOS}}$, we obtain the following first-order differential equation:

$$
k^{\prime}(\operatorname{det} \boldsymbol{F})+\frac{2 k(\operatorname{det} \boldsymbol{F})}{3 e \sqrt{2 e+1}}=\frac{2}{9 B e^{2}} P_{\mathrm{EOS}}(\operatorname{det} \boldsymbol{F})
$$

(with $B$ the bulk modulus at $p=0$ ), solved numerically to obtain $k$.

\section{MD computation of a spherical deformation EOS}

To be consistent with the hypothesis used to set up the nonlinear elasticity, we calculate in this section an equation of state (EOS) through MD simulations by using the prescribed deformation framework introduced by Lafourcade et al. [30] that allows us to apply any deformation path to a MD simulation box. An orthorhombic simulation cell was built using the generalized crystal-cutting method presented by Kroonblawd et al. [14] and the TATB unit cell parameters obtained by Lafourcade et al. [30]. The resulting optimized orthorhombic cell, containing 1824 molecules with length parameters $a=71.542 \AA, b=47.035 \AA$, and $c=120.368 \AA$, was used to calculate the EOS. A second (and larger) supercell, built to study the onset of the buckling-twinning microstructure, consists of a 3D-periodic $14 \boldsymbol{a} \times 22 \boldsymbol{b} \times 1 \boldsymbol{c}$ simulation cell containing 561792 molecules (length $=100.159 \mathrm{~nm}$, width $=103.477 \mathrm{~nm}$, and height $=12.037 \mathrm{~nm}$ ).

The STAMP code [41] was used to perform the MD simulations with the nonpolarizable force field for TATB developed by Bedrov et al. [6] using planar and symmetric rigid molecules. All the interactions were computed with a $13 \AA$ cutoff and the reaction field approximation [42-44] was used to compute the long-range electrostatic interactions instead of using the Ewald sum method. Comparisons have been made in the past [6] and it has been shown that the reaction-field approximation with partial charges scaled by $20 \%$ reproduces accurately the results obtained using the Ewald summation, offering in addition to that a gain in simulation time. This force field has been used in several recent studies to characterize thermomechanical properties of the TATB single crystal such as thermal conductivity [4,5], liquid-transport properties [25], elastic constants $[1,6,7], \gamma$ surfaces $[1,7]$, response under nanoindentation [18], hot spots [45], dislocation core structures [1], response under shock loading [14], and mechanisms

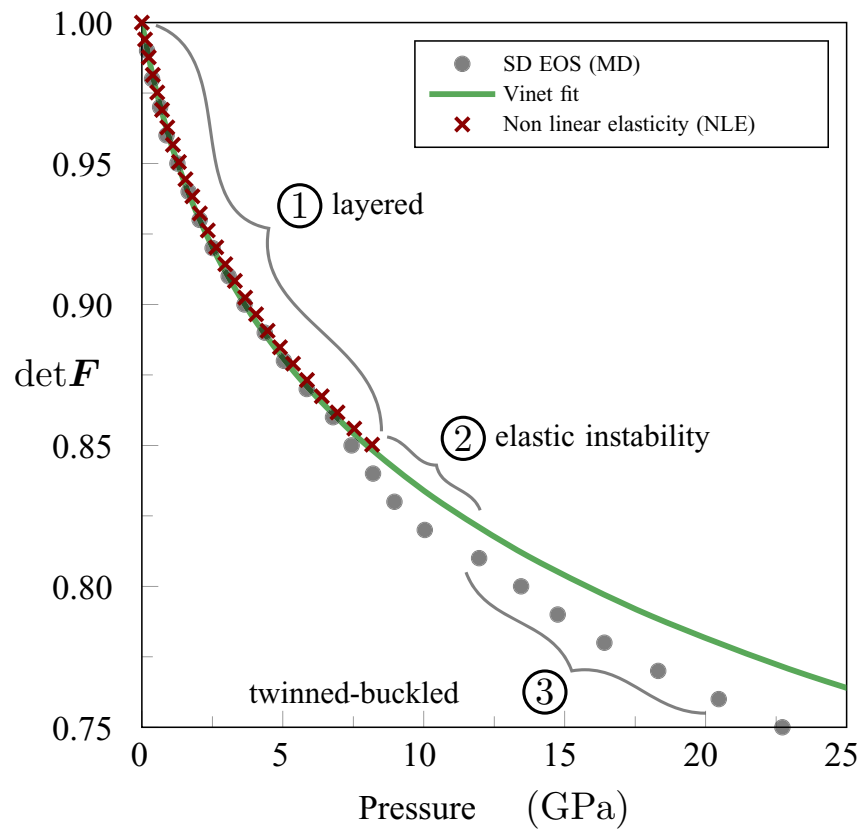

FIG. 2. Spherical deformation EOS (SD-EOS) for TATB single crystal. Gray marks: MD data. Green continuous line: Vinet fit on the compatible part of MD data with $K_{\mathrm{V}}=17.87 \mathrm{GPa}$ and $K_{\mathrm{V}}^{\prime}=12.75$. Red crosses: Equation of state under spherical deformation using the nonlinear elasticity (NLE).

of the irreversible deformation [30]. A Langevin thermostat with a damping constant of $1.0 \mathrm{ps}$ was used and equations of motion were integrated with a $1.0 \mathrm{fs}$ time step for both isochoric-isoenergetic (NVE) and isochoric-isothermal (NVT) simulations. Finally, the rigid-body dynamics was computed by discretization of the equations of motion following a velocity-Verlet scheme [46].

In order to ensure equilibrium in pressure and temperature, TATB cell parameters were obtained using Parrinello-Rahman isostress-isothermal $(N \sigma T)$ simulations that were described in detail in previous studies [1,30]. Then, a 150 ps equilibration trajectory in the NVT ensemble is performed using the optimized cell parameters, which leads to perfect hydrostatic conditions at $p=0$ and $300 \mathrm{~K}$; a spherical deformation was applied to the system through the deformation gradient tensor $\boldsymbol{F}=(\operatorname{det} \boldsymbol{F})^{1 / 3} \boldsymbol{I}$ up to $\operatorname{det} \boldsymbol{F}=0.65$. Thus, in opposition to the hydrostatic pressure loading, the material undergoes the same amount of deformation in the three directions of the space. The resulting pressure-volume curve is reported in Fig. 2 as gray disks. The relationship between pressure and volume exhibits three distinct areas.

The section of the curve denoted 1 corresponds to the first step of the spherical compression (for $0.85<\operatorname{det} \boldsymbol{F}<1.0$ ) during which the TATB single crystal undergoes the deformation while keeping its layered structure. For lower volumes $(0.80<\operatorname{det} \boldsymbol{F}<0.85$; section 2$)$ we observe an instability leading to the onset of a complex microstructure (stable up to $\operatorname{det} \boldsymbol{F}<0.80$ ) where twinning and buckling are mixed [30] (section 3). This last microstructure, stable under pressure, has pressure and volume compatible with a Vinet EOS but with different parameters. This particular state will be discussed in Sec. IV. 
TABLE I. Material parameters used for the TATB single-crystal EOS computation under spherical deformation.

\begin{tabular}{cc}
\hline \hline Density in $\mathrm{g} / \mathrm{cm}^{3}$, after Lafourcade et al. $[1]$ \\
\hline$\rho_{0}$ & 1.93 \\
Stiffness in GPa, after Lafourcade et al. $[1]$ & 49.88 \\
$C_{11}$ & 50.07 \\
$C_{22}$ & 24.66 \\
$C_{33}$ & 2.32 \\
$C_{44}$ & 1.92 \\
$C_{55}$ & 25.22 \\
$C_{66}$ & 11.41 \\
$C_{12}$ & 2.39 \\
$C_{13}$ & 4.30 \\
$C_{23}$ & $17.87 \mathrm{GPa}$ \\
& Vinet EOS (this work) \\
$K_{\mathrm{V}}$ & 12.75 \\
$K_{\mathrm{V}}^{\prime}$ &
\end{tabular}

A fit with a Vinet equation of state is performed for $0.85<$ $\operatorname{det} \boldsymbol{F}<1.0$. For this purpose, the bulk modulus $K_{\mathrm{V}}$ has been computed from the elastic tensor obtained at $(300 \mathrm{~K}, 0 \mathrm{GPa})$ through MD simulations [1] and the fit performed by looking for an appropriate value of $K_{\mathrm{V}}^{\prime}$. The Vinet curve is represented as a green continuous line in Fig. 2 with values for $K_{\mathrm{V}}$ and $K_{\mathrm{V}}^{\prime}$ given in Table I. The corresponding pressure-volume curve validates the nonlinear elasticity formalism [Eqs. (13) to (16)] with the parameters listed in Table I, down to $\operatorname{det} \boldsymbol{F}=0.85$, plotted as red crosses in Fig. 2.

For higher compressions, an instability involving both an elastic buckling and a twinning of the materials leads to a complex microstructure, the modeling of which implies additional deformation mechanisms, described in the next section.

\section{PHASE FIELD BY REACTION PATHWAY FORMALISM FOR IRREVERSIBLE DEFORMATION}

The buckling instability, observed when the TATB single crystal is compressed along a direction lying in its basal plane, induces strong shear stresses. For directions of compression parallel to [010], this stress leads to a twinning, an irreversible nonsymmorphic transformation due to the existence of a second energy minimum in addition to the strain-free one in the so-called "twinning-energy landscape" [30]. This landscape is calculated in MD by imposing a deformation gradient $\boldsymbol{F}=\alpha \boldsymbol{e} \times \boldsymbol{n}+\boldsymbol{I}$, with $\alpha$ a scalar, $\boldsymbol{e}$ a direction in the basal plane, and $\boldsymbol{n}$ the basal plane normal. In Fig. 3 are reported multiple energy profiles along different homogeneous shear directions associated with different $\boldsymbol{e}$ vectors. The yellow line corresponds to a deformation with $\boldsymbol{e}=\boldsymbol{a}$, while $\boldsymbol{e} \perp \boldsymbol{a}=^{\perp}$ $\boldsymbol{a} \in(001)$ is traduced by the black line, where $\boldsymbol{a}$ is one of the TATB unit cell basal plane vectors. Finally, intermediate energy profiles, represented by colors smoothly varying from yellow to black, correspond to intermediate shear deformations. While the first line has a single minimum, the second one has two minima of the same energies (denoted in the following $\epsilon$ and $\epsilon^{T}$ ), these two minima being related by the twinning transformation.

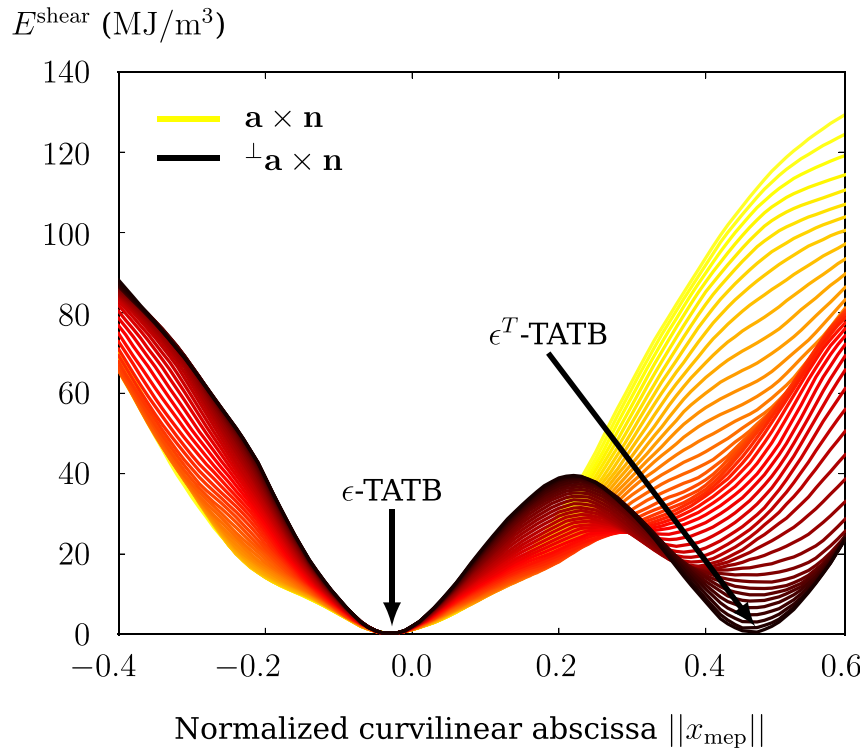

FIG. 3. Energy profiles of the twinning-energy landscape along different directions passing by the $\epsilon$-TATB phase strain-free energy minimum from MD simulations.

A method developed for martensitic transformations $[31,32]$ and applied to pressure-induced phase transformation of iron [33] has been used to take into account the shearinduced twinning phase transition in the TATB single crystal. The aim of this section is to detail the PF-RP method adapted to TATB in order to perform EFG simulations with a coupling between nonlinear elasticity, twinning phase transition, and buckling instability.

\section{A. Evolution of phase transitions}

A linear kinetic equation that links the transformational distortion rate $\dot{\boldsymbol{F}}_{\mathrm{t}}$ to the displacive driving forces $\boldsymbol{X}_{\mathrm{t}}$ is adopted:

$$
v \dot{\boldsymbol{F}}_{\mathrm{t}}=\boldsymbol{X}_{\mathrm{t}},
$$

where $v>0$ is a viscosity-like parameter ( $v=0$ corresponding to a nondissipative evolution). This relation ensures a positive dissipation, due only to twinning [see Eq. (7)]: $\mathcal{D}=\boldsymbol{X}_{\mathrm{t}}: \dot{\boldsymbol{F}}_{\mathrm{t}} \geqslant 0$.

\section{B. Reaction pathway and inelastic energy landscape}

In previous models based on PF-RP [31-33], numerous pathways, connected in a open tree, were used, each pathway extremity corresponding to a stable or metastable phase. In the present study, only two stable states are known (the initial lattice and its twinned counterpart). We therefore need only one pathway to represent the twin transformation $\epsilon \leftrightarrow \epsilon^{T}$. The reaction pathway formalism as well as the associated inelastic energy landscape for one pathway is thus derived in the following.

\section{The $\epsilon \leftrightarrow \epsilon^{T}$ nonsymmorphic twinning mechanism}

Let us consider a centrosymmetric unit cell of TATB containing two molecules with lengths $\boldsymbol{a}, \boldsymbol{b}$, and $\boldsymbol{c}$, 
out-of-plane angles $\alpha, \beta$, and basal plane angle $\gamma$ between $\boldsymbol{a}$ and $\boldsymbol{b}$ lattice vectors. Applying a pure shear to the basal plane while keeping the interlayer distance constant leads to a perfect nonsymmorphic twinning of the unit cell [30], with a modification of the two out-of-plane angles only: $\alpha^{\prime}=$ $180.0-\alpha$ and $\beta^{\prime}=180.0-\beta$, where the 'symbol denotes the angles of the new twinned lattice. By taking $\boldsymbol{H}_{\boldsymbol{\epsilon}}$ and $\boldsymbol{H}_{\boldsymbol{\epsilon}^{T}}$ the second-rank transformation tensors from fractional to crystallographic coordinates of $\epsilon$ and $\epsilon^{T}$ phases, respectively, the right Cauchy-Green strain tensor for this transformation reads

$$
\begin{gathered}
\boldsymbol{C}_{\mathrm{twin}}=\boldsymbol{H}_{\boldsymbol{\epsilon}}^{-\boldsymbol{T}} \cdot \boldsymbol{H}_{\boldsymbol{\epsilon}^{\boldsymbol{T}}}^{\boldsymbol{T}} \cdot \boldsymbol{H}_{\boldsymbol{\epsilon}^{T}} \cdot \boldsymbol{H}_{\boldsymbol{\epsilon}}^{-\mathbf{1}}, \\
\boldsymbol{C}_{\mathrm{twin}}=\left[\begin{array}{ccc}
1 & 0 & -\frac{2 \cos \beta}{n_{3}} \\
0 & 1 & -\frac{2 n_{2}}{n_{3}} \\
-\frac{2 \cos \beta}{n_{3}} & -\frac{2 n_{2}}{n_{3}} & \frac{n_{3}^{2}+\left(4 \cos ^{2} \beta+4 n_{2}^{2}\right)}{n_{3}^{2}}
\end{array}\right],
\end{gathered}
$$

where $n_{2}$ and $n_{3}$ are two functions or out-of-plane angles that read $n_{2}=(\cos \alpha-\cos \gamma \cos \beta) / \sin \gamma$ and $n_{3}=$ $\sqrt{\sin ^{2} \beta-n_{2}^{2}}$. This transformation consists of an elongation along the $c$ direction and a pure shear in the basal plane.

\section{Inelastic energy landscape}

The inelastic energy $\psi_{\mathrm{t}}$ related to the twin transformation is a function of the transformational Cauchy-Green strain tensor $\boldsymbol{C}_{\mathrm{t}}=\boldsymbol{F}_{\mathrm{t}}^{T} \cdot \boldsymbol{F}_{\mathrm{t}}$, i.e.,

$$
\psi_{\mathrm{t}} \doteq \psi_{\mathrm{t}}\left(\boldsymbol{C}_{\mathrm{t}}\right)
$$

Two stable states (the initial state, $\boldsymbol{C}_{\mathrm{t}}=\boldsymbol{I}$, and the twinned one characterized by the Cauchy-Green tensor $\boldsymbol{C}_{\mathrm{twin}}$ ) are used to define a pathway through a linear interpolation of starting and ending phases:

$$
\hat{\boldsymbol{C}}_{\mathrm{t}}(s)=\left(1-\frac{s}{s_{\mathrm{twin}}}\right) \boldsymbol{I}+\frac{s}{s_{\mathrm{twin}}} \boldsymbol{C}_{\mathrm{twin}}
$$

with $s$ (between 0 and $s_{\text {twin }}=\left|\boldsymbol{C}_{\text {twin }}-\boldsymbol{I}\right|$ ) the curvilinear abscissa along the pathway. This abscissa is defined for any $\boldsymbol{C}_{\mathrm{t}}$ by a projection operation:

$$
s\left(\boldsymbol{C}_{\mathrm{t}}\right)=\left\{0, \boldsymbol{D}:\left(\boldsymbol{C}_{\mathrm{t}}-\boldsymbol{I}\right), s_{\mathrm{twin}}\right\},
$$

where $\boldsymbol{D}=\left(\boldsymbol{C}_{\text {twin }}-\boldsymbol{I}\right) / s_{\text {twin }}$ is the normalized pathway direction, and $\left\{0, s, s_{\text {twin }}\right\}$ means that the value of $s$ is clamped to $\left[0, s_{\text {twin }}\right]$.

We define the inelastic energy $\psi_{\mathrm{t}}\left(\boldsymbol{C}_{\mathrm{t}}\right)$ as the sum of a function of $s$ only and a term proportional to the distance to the pathway:

$$
\psi_{\mathrm{t}}\left(\boldsymbol{C}_{\mathrm{t}}\right)=\hat{\psi}_{\mathrm{t}}\left[s\left(\boldsymbol{C}_{\mathrm{t}}\right)\right]+\sigma_{\mathrm{pw}} d\left(\boldsymbol{C}_{\mathrm{t}}\right),
$$

where $d\left(\boldsymbol{C}_{\mathrm{t}}\right)=\left|\boldsymbol{C}_{\mathrm{t}}-\hat{\boldsymbol{C}}_{\mathrm{t}}\left[s\left(\boldsymbol{C}_{\mathrm{t}}\right)\right]\right|$ and $\sigma_{\mathrm{pw}}$ is a phenomenological parameter associated with the stress required to take the phase away from a pathway. Since $s$ is defined as the abscissa that minimizes $d\left(\boldsymbol{C}_{\mathrm{t}}\right)$ [32], $d$ is the distance to the pathway, represented as a linear segment. The inelastic energy along the pathway $\hat{\psi}_{\mathrm{t}}(s)$ can be postulated or deduced from classical MD energy calculations and will be detailed in Sec. III C.
TABLE II. Material parameters for the PF-RP formalism used for shear-induced twinning transformation in TATB.

\begin{tabular}{lcr}
\hline \hline & Cauchy-Green components (no units) & \\
\hline $\boldsymbol{C}_{\mathrm{t}}^{11}$ & 1.0 & 1.0 \\
$\boldsymbol{C}_{\mathrm{t}}^{22}$ & 1.0 & 1.0 \\
$\boldsymbol{C}_{\mathrm{t}}^{33}$ & 1.0 & 1.272 \\
$\boldsymbol{C}_{\mathrm{t}}^{13}$ & 0.0 & -0.055 \\
$\boldsymbol{C}_{\mathrm{t}}^{23}$ & 0.0 & 0.519 \\
& Energy landscape parameters & \\
$\tau$ & & 0.2 \\
$\sigma(\mathrm{MPa})$ & -85.0 & \\
$v(\mathrm{Pas})$ & & 0.13 \\
$\sigma_{\mathrm{pw}}(\mathrm{GPa})$ & 10.0 \\
\hline \hline
\end{tabular}

\section{Transformational driving forces}

Inelastic driving forces due to transformational distortions are deduced from the derivative of the inelastic potential $\psi_{\mathrm{t}}$ with respect to the transformational part of the deformation gradient tensor $\boldsymbol{F}_{\mathrm{t}}$ :

$$
\frac{\partial \psi_{\mathrm{t}}}{\partial \boldsymbol{F}_{\mathrm{t}}}=2 \boldsymbol{F}_{\mathrm{t}} \cdot \frac{\partial \psi_{\mathrm{t}}\left(\boldsymbol{C}_{\mathrm{t}}\right)}{\partial \boldsymbol{C}_{\mathrm{t}}} .
$$

According to Eq. (23), the last derivation term reads

$$
\frac{\partial \psi_{\mathrm{t}}\left(\boldsymbol{C}_{\mathrm{t}}\right)}{\partial \boldsymbol{C}_{\mathrm{t}}}=\frac{\partial \hat{\psi}_{\mathrm{t}}\left(s\left(\boldsymbol{C}_{\mathrm{t}}\right)\right)}{\partial s\left(\boldsymbol{C}_{\mathrm{t}}\right)} \boldsymbol{D}+\sigma_{\mathrm{pw}} \boldsymbol{N},
$$

with $N$ the normal to the pathway pointing towards $\boldsymbol{C}_{\mathrm{t}}$ defined as $\boldsymbol{N}\left(\boldsymbol{C}_{\mathrm{t}}\right)=\left\{\boldsymbol{C}_{\mathrm{t}}-\hat{\boldsymbol{C}}_{\mathrm{t}}\left[s\left(\boldsymbol{C}_{\mathrm{t}}\right)\right]\right\} / d\left(\boldsymbol{C}_{\mathrm{t}}\right)$.

Inserting Eqs. (24) and (25) into Eq. (26) leads to the final expression of the transformational driving forces:

$$
\begin{aligned}
\nu \dot{\boldsymbol{F}}_{\mathrm{t}}= & \boldsymbol{C}_{\mathrm{e}} \cdot\left[\mathbb{D}(\operatorname{det} \boldsymbol{F}): \boldsymbol{E}_{\mathrm{e}}\right] \cdot \boldsymbol{F}_{\mathrm{t}}^{-T} \\
& -2 \rho_{0} \boldsymbol{F}_{\mathrm{t}}\left(\frac{\partial \hat{\psi}_{\mathrm{t}}\left(s\left(\boldsymbol{C}_{\mathrm{t}}\right)\right)}{\partial s\left(\boldsymbol{C}_{\mathrm{t}}\right)} \boldsymbol{D}+\sigma \boldsymbol{N}\right) .
\end{aligned}
$$

\section{Shear-induced twinning in TATB single crystal with PF-RP}

Lafourcade et al. [30] computed the twinning-energy landscape under pure shear as well as the minimum energy path that links the two stable phases. After differentiating this energy landscape with respect to the appropriate deformation gradient tensor, they obtained an activation von Mises stress of around $150 \mathrm{MPa}$. To reproduce this threshold, we choose for the parametrization of $\hat{\psi}_{\mathrm{t}}(s)$ a third-order polynomial function

$$
\hat{\psi}_{\mathrm{t}}(\bar{s})=\bar{s} \sigma(\bar{s}-1)(1+\tau \bar{s}),
$$

where $\bar{s}=s / s_{\text {twin }}$, and $\sigma$ and $\tau$ are two parameters used to fit the threshold of $150 \mathrm{MPa}$ for the twinning and the untwinning transformation. It is straightforward that when $s$ is equal to its two bounds the inelastic energy equals zero, consistently with the same equilibrium properties of the two phases. Values of the threshold, Cauchy-Green strain components, and model parameters concerning the two stable and twinned phases are given in Table II.

In order to illustrate the inelastic potential computed on the reaction pathway for the twinning transformation in TATB, we 


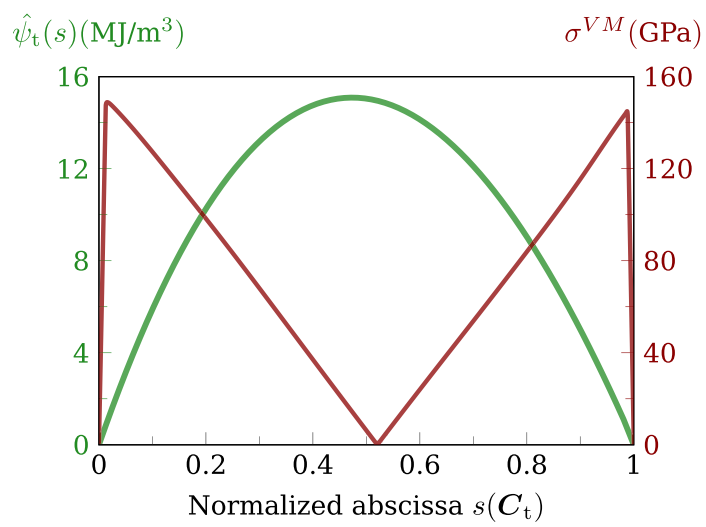

FIG. 4. Green line: Inelastic energy on the single-reaction pathway. Red line: The von Mises stress for a deformation along the reaction pathway.

applied an arbitrary isochoric shear deformation corresponding to a Cauchy-Green strain tensor $\boldsymbol{C}_{\mathrm{t}}=x\left(\boldsymbol{C}_{\mathrm{t}}-\boldsymbol{I}\right)+\boldsymbol{I}$ with $x \in[0,1]$, with a very low viscosity so that the thermodynamic force $\boldsymbol{X}_{\mathrm{t}}=\mathbf{0}$. The resulting von Mises stress profile as well as the inelastic energy landscape are provided in Fig. 4.

\section{ONE-TO-ONE COMPARISONS WITH MOLECULAR DYNAMICS SIMULATIONS}

This section is dedicated to one-to-one comparisons between MD and continuum mechanics simulations in order to validate the constitutive law presented in the preceding sections. We first present the computational framework of both MD simulations and the element-free Galerkin formulation used to challenge our constitutive law. Then, we present comparisons for uniaxial and spherical compressions of a TATB single crystal.

\section{A. Computational framework}

The response of the single crystal under different types of loading was characterized by MD simulations using the same system size as presented in Sec. II D for the EOS computation as well as the same computational framework (i.e., a system with lengths of $100.159 \mathrm{~nm}, 103.477 \mathrm{~nm}$, and $12.037 \mathrm{~nm}$ per side). For each MD simulation discussed hereafter, a $150 \mathrm{ps}$ trajectory was calculated in the $N V T$ ensemble in order to reach equilibrium in temperature and hydrostatic pressure (i.e., $300 \mathrm{~K}, 0 \mathrm{GPa}$ ). The final configuration of this trajectory is then considered as the initial one for subsequent MD simulations (i.e., $t=0$ ). Then, the system is dynamically strained using the prescribed deformation framework introduced in [30], without any thermostat.

For the continuum-level simulations, an element-free Galerkin (EFG) least-squares formulation code [47] was used in a 3D Lagrangian framework, with an explicit time integration. Both a nonlinear elasticity framework (presented in Sec. II) and the PF-RP formalism (see Sec. III) were coupled in order to take into account TATB elastic anisotropy in pressure as well as the shear-induced twinning mechanism. For the simulation of the TATB single crystal behavior under different mechanical loadings, the material parameters (listed in Tables I and II) that have been computed through MD simulations were used as inputs of the constitutive law. The simulated material is a plate of TATB single crystals with the same dimensions as the MD simulation sample containing $7623(33 \times 33 \times 7)$ elements with 3D-periodic boundary conditions. For each deformation investigated in the following, the final simulation time $t_{f}$ was chosen to be the same as in $\mathrm{MD}$ in order to be able to perform one-to-one comparisons.

\section{B. Uniaxial compressions in the (001) plane}

When a uniaxial compression is applied in a direction that lies in the basal plane of the TATB single crystal, a buckling of the molecular layers is observed, leading to a chevronlike microstructure with interfaces nearly perpendicular to the direction of loading. In the following, the same uniaxial compressions as in [30] were prescribed but with an orthorhombic supercell, thus with different boundary conditions. In the same way, we define an orthonormal basis $(\boldsymbol{x}, \boldsymbol{y}, \boldsymbol{z})$ with $\boldsymbol{x}$ parallel to $\boldsymbol{a}, \boldsymbol{y}$ parallel to $\boldsymbol{b}, \boldsymbol{z}$ parallel to $\boldsymbol{c}$ (i.e., normal to the molecular layers $)$, and the direction of compression $\boldsymbol{m}=(\cos \theta, \sin \theta, 0)$ with $\theta \in[0,2 \pi]$ [see Fig. 5(a)].

Three directions of compression were investigated at a constant strain rate of $4 \times 10^{8} \mathrm{~s}^{-1}$ with a compression ratio $R=10 \%$. The TATB orthorhombic supercell was strained along the direction $\boldsymbol{m}$ with $\theta=0^{\circ}, 75^{\circ}$, and $90^{\circ}$. In Fig. 5(c) are shown the snapshots of the three different MD simulations with $\theta=0^{\circ}, 75^{\circ}$, and $90^{\circ}$ aligned from left to right at the end of the dynamic loading, i.e., at $R=10 \%$. A color coding has been defined to ease the simulation analysis. Since buckling involves large shear of the basal plane due to the low friction between molecular layers, the Green-Lagrange strain tensor components that are the most affected are $E_{13}$ and $E_{23}$. The couple $\left(E_{13}, E_{23}\right)$ is computed for each molecule leading to a density map $d\left(E_{13}, E_{23}\right)$, such that the number of molecules with deformation $\left(E_{13} \pm \Delta / 2, E_{13} \pm \Delta / 2\right)$ is given by $d\left(E_{13}, E_{23}\right) \times \Delta^{2}$ for a small deformation amplitude $\Delta$. After extraction of the maxima and lumping the remaining density to the nearest maximum, Lafourcade et al. [30] obtained a simplified histogram as a list of maxima, each one associated with a fraction of the system affected by the neighboring strain. The deformation signatures fall in the vicinity of the deepest energy wells in the twinning-energy landscape presented in Fig. 3. This means that when a compression with $\theta=0^{\circ}$ is applied to the sample, the maxima are located on the $E_{13}$ axis and centered around the origin, due to the quadratic elastic energy well (see Fig. 3, yellow line). On the other hand, when a compression with $\theta=90^{\circ}$ is considered, the maxima are located on the $E_{23}$ axis but in the vicinity of the two energy wells (see Fig. 3, black line). These two configurations represent some sort of bounds in the resulting microstructure, which means that for intermediate directions of compression, $E_{13}$ and $E_{23}$ will live within these ranges. By considering a centered blue-black-red RGB color scale between -0.2 and 0.2 for $E_{13}$, a noncentered black-green RGB color scale between 0 and 0.25 for $E_{23}$, and by screening the RGB values of the two perpendicular color scales, we obtain the twodimensional color coding presented in Fig. 5(b). Thus, blue and red correspond to negative and positive buckling, black 
(a) Material loading configuration

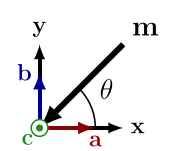

(b)
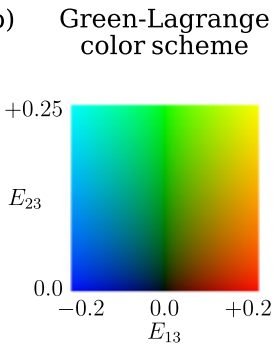

(c)

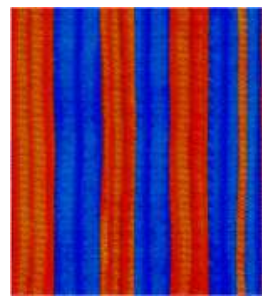

(d)

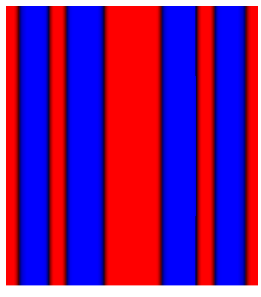

MD simulations

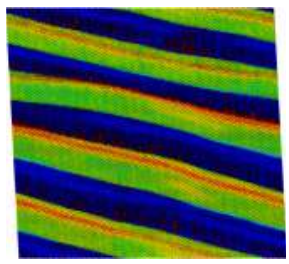

EFG simulations

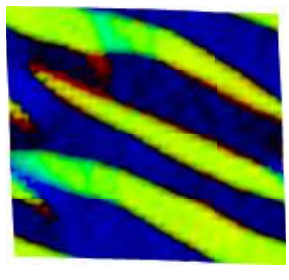

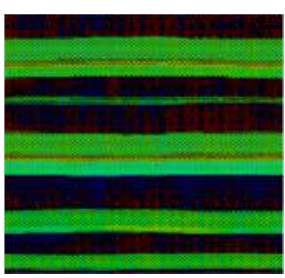

(e)

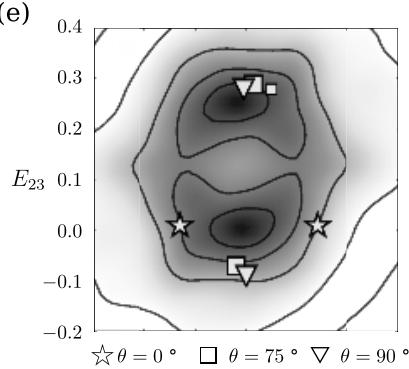

(f)

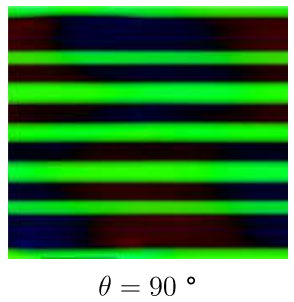

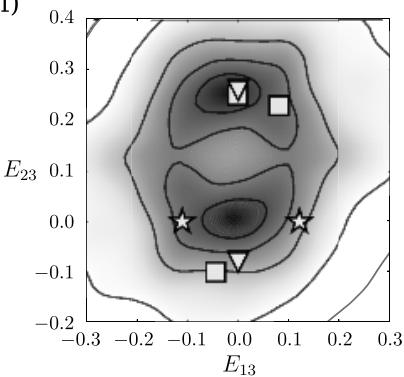

FIG. 5. (a) Orientation of loading with respect to the single-crystal Bravais vectors. (b) Green-Lagrange color scheme. (c) Snapshots of the three MD simulations. (d) Snapshots of the three EFG simulations. (e) and (f) Deformation histograms reported on the twinning-energy landscape for MD and EFG simulations, respectively.

to null $x z$ and $y z$ shear components, green to shear-induced twinning, while yellow and cyan represent compositions of buckling and twinning.

When $\theta=0^{\circ}$ and $90^{\circ}$, pure buckling and pure twinning are activated, respectively. The case $\theta=75^{\circ}$ involves a composition of buckling and twinning but with a dominance for the latter. In the three cases, small additional deformation bands appear and are associated with localized basal gliding of molecular layers due to the arrangement of molecules at the chevrons interfaces. However, this phenomenon is limited to a few planes and we consider the twinning-buckling to be predominant. The deformation histograms [Fig. 5(e)] are comparable with the ones presented by Lafourcade et al. [30] with nonorthogonal boundary conditions, with the most of the deformation histograms in the vicinity of the two deepest energy wells that span the energy landscape.

Final snapshots of the three different dynamic compressions (i.e., with $\theta=0^{\circ}, 75^{\circ}$, and $90^{\circ}$ ) performed with our model are presented in Figs. 5(c) and 5(d) with the same color coding as for MD simulations. These simulations include the nonlinear elasticity and PF-RP formalisms presented in Sec. II and III. A very good agreement between both methods is found in terms of intensity of deformation components and buckling and twinning microstructures. Additionally, deformation histograms have also been reported on the twinning-energy landscape in Fig. 5(f) in order to compare with MD simulations. The histograms of MD and EFG simulations are found to be very similar, with however small differences for $\theta=75^{\circ}$, observed both in the deformation histograms and the microstructure. These deviations are due to the incapacity of the continuum model to reproduce very localized features such as local molecular reorientations or local shift of molecules at the interface between different buckled regions due to the change of resolution, inherent to the proposed model.

\section{Spherical compression}

The EOS computed through MD simulations under spherical compression (hereafter termed "spherical EOS") leads to an elastic instability at approximately $V / V_{0}=\operatorname{det} \boldsymbol{F}=0.85$ (see Fig. 2). The aim of this section is to compare the microstructure predicted by the constitutive law with the one obtained through MD calculations in terms of Green-Lagrange deformation components and deformation histograms.

Due to the high anisotropy of the TATB single crystal, a spherical compression induces a nonhydrostatic stress large enough to induce a buckling-twinning transition. Using the same EFG simulation box as in the uniaxial compressions case, we performed a spherical compression at $V / V_{0}=0.80$, equivalent to a longitudinal strain of $7.2 \%$ in each direction of the space, at a strain rate of $4 \times 10^{8} \mathrm{~s}^{-1}$. A snapshot corresponding to the end of the dynamic loading of the MD simulation is shown in Fig. 6(a), as well as the matching deformation histograms in Fig. 6(c). The color scheme used is the same as in Fig. 5. The tessellation of the sample is characterized by well-defined deformation signatures, with pure negative and positive buckling as well as composition with twinning. However the deformation histogram is centered on the zero deformation minimum, reflecting strain equilibrium. Similarly to the uniaxial compressions, small additional deformation bands can be observed in the MD microstructure and are due to basal plane gliding to accommodate the molecular stacking at the interfaces.

The same spherical compression was performed using the EFG code and the obtained microstructure and histograms are reported in Fig. 6 [panels (b) and (d)]. Except for very narrow deformation bands caused by basal gliding, the predicted tessellation is comparable to the one obtained by MD simulations. Additionally, a very good agreement is found for the deformation histograms which are also centered on the zero deformation energy minimum. 
(a)

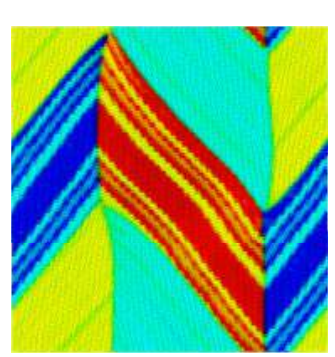

(c)

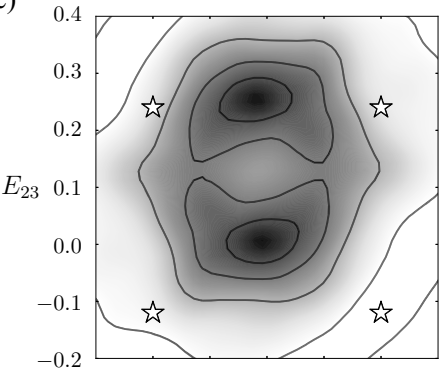

(b)

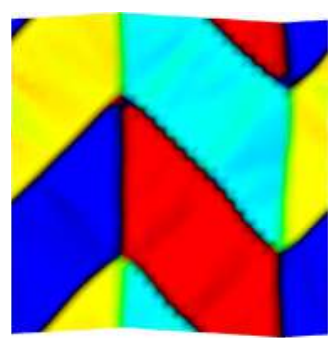

(d)

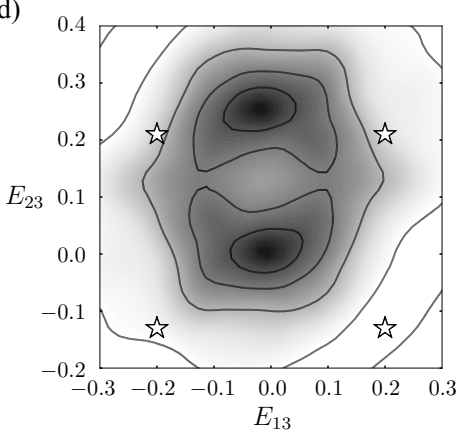

FIG. 6. (a) Snapshot of the MD simulation. (b) Snapshot of the EFG simulation. (c) and (d) Deformation histograms reported on the twinning-energy landscape for MD and EFG simulations, respectively.

\section{UPSCALING CASE: TATB POLYCRYSTAL UNDER WEAK SHOCK LOADING}

\section{A. Simulation parameters}

The present section is dedicated to an upscaling case: the TATB polycrystal under shock loading. Indeed, classical molecular dynamics simulations do not allow simulating TATB polycrystals of reasonable size. Thus, we take advantage of the constitutive law hereby presented to study the behavior of a TATB polycrystal under weak shock loading.

The materials parameters provided in Tables I and II were used. In order to create the 2D periodic polycrystal, we first generate a list of 100 randomly distributed seeds in the simulation cell, each one being assigned with an orientation drawn at random in the unit sphere. Finally, a Voronoï tessellation is performed, leading to the final TATB polycrystal with a total number of 49500000 elements $(150 \times 150 \times 2200)$, equivalent to dimensions of $0.46 \mu \mathrm{m} \times$ $0.46 \mu \mathrm{m} \times 6.71 \mu \mathrm{m}$. The obtained microstructure is shown in Fig. 7(c), where the grains are colored according to their seed number.

The shock simulation on the TATB polycrystal is performed by applying a velocity $U_{\mathrm{p}}=150 \mathrm{~ms}^{-1}$ to the $z^{-}$face of the sample in the $z$ direction, corresponding to its longest length. The choice of a relatively low piston velocity has been made in order to generate moderate pressures, ensuring that only elastic and transformational regimes of the TATB single crystal are applied. This is consistent with the fact that our constitutive law does not include the plastic behavior of the TATB single crystal by means of nonbasal dislocations nucleation as introduced by Lafourcade et al. [30].

\section{B. Shock-induced twinning-buckling deformation}

\section{General behavior}

A snapshot of the simulation cell is represented in Fig. 7(a) and was captured just before the elastic wave reaches the opposite face of the sample. The color code of Fig. 5 is used to represent the field of two components of the Green-Lagrange strain ( $E_{13}$ and $\left.E_{23}\right)$, defined in the crystal coordinates: red and blue areas correspond to pure buckling, green to twinning, and black to small pure shear strain areas. Additionally, yellow and cyan zones correspond to a composition of twinning and buckling, as observed in the preceding sections.

Most of the sample is affected by relatively homogeneous strain, which is consistent with an elastic deformation of the grains. However, despite the very low velocity of the impact loading, some localized oscillations of the deformation appear behind the elastic-wave shock front. These bands are associated with alternating shear deformation for grain orientations such that the basal plane is parallel to the shock direction. This buckling instability nucleates for a moderate compressive stress (below $5 \mathrm{GPa}$ ). The higher stresses in the sample come from grain boundaries with strong misorientation and not from the buckling instability that could be described as an accommodation mechanism, driven by elasticity and thus reversible.

One can see that the entire sample is subjected to this complex behavior and various areas of pure buckling, pure twinning, and compositions can be observed. By computing the projection of the transformational part of the CauchyGreen strain tensor onto the reaction pathway considered in our PF-RP model, we are able to identify the local phase within the TATB single crystals, i.e., strain-free $(\epsilon-$ TATB) or twinned $\left(\epsilon^{T}\right.$-TATB $)$ phases. This is represented in Fig. 7(b), where the local field varies from 0 (transparent) to 1 (opaque, orange) for $\epsilon$ and twinned $\epsilon^{T}$ phases, respectively. In this image, one can see the layered structure of twins that passes through some TATB grains. Right behind the shock front, the density of twins is quite low whereas it increases with the distance from it. This reflects the viscous evolution of $\boldsymbol{F}_{\mathrm{t}}$ used in the present model, which, in addition to the inertia needed to accompany the deformation and material rotation, induces smooth evolution for the twinning transformation.

One can see that not all ripples are associated with the twinned phase of TATB. Indeed, although large shear strain is applied in the basal plane of some grains, if the buckling shear is not along the pathway (i.e., the deformation produces no gliding in the twinning direction) then only buckling is obtained. In Fig. 7(d), we focus on a part of the polycrystal where multiple grains are subjected to large strains, without the appearance of twinning transformation. Indeed, we can see that some grains (e.g., black, cyan, and green grains) are subjected to homogeneous deformation. The black one has null $E_{13}$ and $E_{23}$ components while the cyan and green ones have undergone composition of directional shear in their basal plane. On other grains, very fine layered microstructure are obtained and correspond to buckling (blue-red) and twinningbuckling (cyan-black) areas. Some of these very oriented features are also curved, emphasizing a gradient of directions for the eigenstress, due to grain interaction. This fine and 
(a)

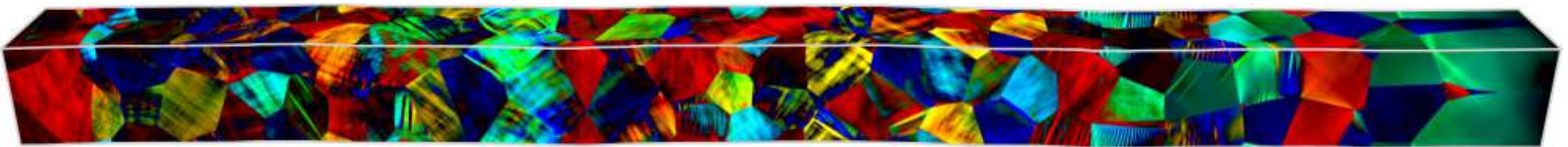

(b)

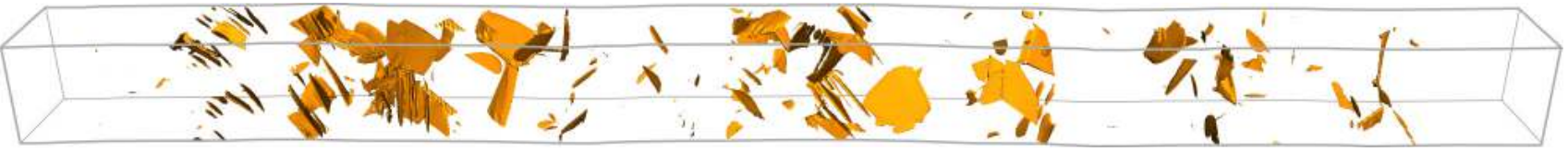

(c)

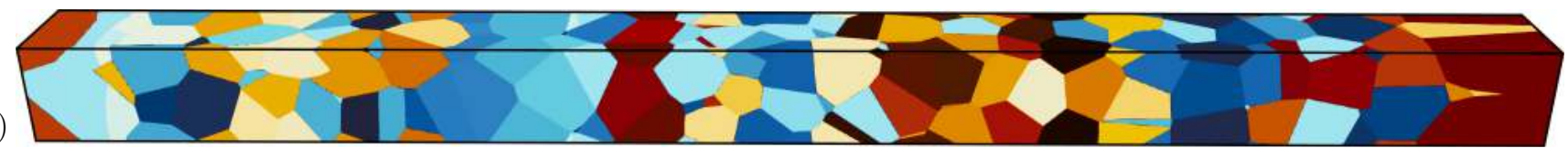

(d)

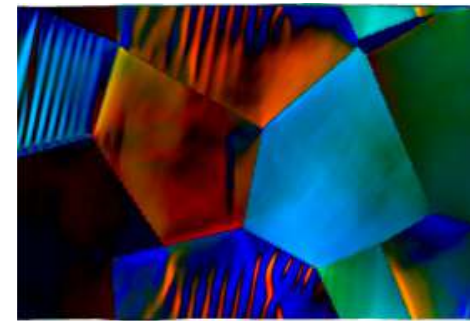

(e)

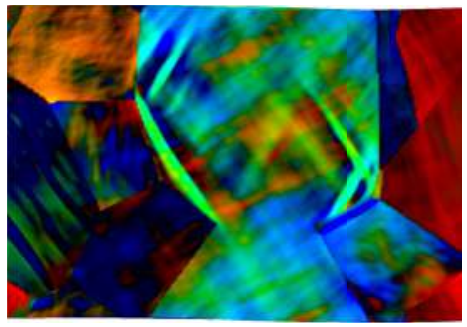

FIG. 7. Snapshot of the shock-loading simulation. (a) Local deformation using the Green-Lagrange color scheme. (b) Representation of the multiple twins nucleated during the shock loading. (c) Distribution of the 100 different grains in the polycrystal. (d) Focus on a few grains with a fine buckling microstructure. (e) Focus on a few grains where multiple orientations of twinning-buckling are observed.

smooth microstructure highlights the absence of friction on the used mesh.

\section{In-grain non-uniaxial response}

Finally, some grains exhibit twin bands with a small curvature or even multiple orientations ("tweedlike" pattern) as shown in Fig. 7(e). In this snapshot, the central grain exhibits a composition of multiple twinning-buckling orientations. Indeed, we can distinguish different orientations for (green) thin deformation bands that actually are twins. Also, there are underlying red and blue patterns that correspond to a pure buckling mechanism. This behavior reflects nearly hydrostatic stresses due to the interactions with the grain's neighbors, leading to this complex microstructure that could be similar to the spherical compression results presented in Sec. III.

\section{CONCLUSIONS}

In this work, we present a molecular-dynamics-informed constitutive law for the mechanical behavior of the highly anisotropic energetic molecular crystal 1,3,5-triamino-2,4,6trinitrobenzene. The constitutive law contains two main features: the first one is the definition of a pressure-dependent nonlinear elasticity formalism to model the strong elastic anisotropy and a cold equation of state. The second feature of the present constitutive law is the phase field by reaction pathways (PF-RP) formalism, introduced to model the twinning mechanism. The PF-RP is defined through a strain pathway to which is associated an "inelastic" energy, taken into account in the constitutive law. By applying a deformation along this pathway, the PF-RP parameters are adjusted to reproduce the stress levels obtained in the MD simulations.

Various problems are then chosen to test the constitutive law. Uniaxial compressions along various directions in the TATB single crystal basal plane are performed with both simulation methods and the results are discussed in detail. A very good agreement is obtained on the one-to-one comparisons and both microstructure and deformation histograms from MD are well reproduced using the mesoscopic model. Then, the behavior of the single crystal is studied under spherical compression. Again, a very good agreement between both techniques is observed with the exact reproduction of both microstructure and deformation histograms. The only difference between microscopic and mesoscopic simulations emerges from the ability of the material to accommodate the deformation at the interfaces of the twinning-buckling patterns through local slip in the basal plane, which has not been including in the present model. The role of temperature has not been extensively discussed in this work since during the molecular dynamics simulations, no significant increase in temperature was observed. This is completely consistent with the mechanical response of TATB, i.e., elastic twinningbuckling deformation without dislocation nucleation and thus no dissipation in the system due to plastic activity. No crystal plasticity formalism was included in the present constitutive law, which is therefore not able to represent temperature effects.

Finally, a shock-loading simulation $\left(U_{\mathrm{p}}=150 \mathrm{~ms}^{-1}\right)$ on a TATB polycrystal containing 100 grains is performed with sizes nonreachable by using MD simulations. We observe 
that behind the shock front, twin bands are generated in various grains with sometimes multiple orientations within the same grain. These results show that even for low-velocity shocks, the twinning-buckling mechanism is activated, leading to a strong modification of the initial polycrystalline microstructure.
[1] P. Lafourcade, C. Denoual, and J.-B. Maillet, J. Phys. Chem. C 121, 7442 (2017).

[2] H. H. Cady and A. C. Larson, Acta Crystallogr. 18, 485 (1965).

[3] T. Plisson, N. Pineau, G. Weck, E. Bruneton, N. Guignot, and P. Loubeyre, J. Appl. Phys. 122, 235901 (2017).

[4] M. P. Kroonblawd and T. D. Sewell, J. Chem. Phys. 139, 074503 (2013).

[5] M. P. Kroonblawd and T. D. Sewell, J. Chem. Phys. 141, 184501 (2014).

[6] D. Bedrov, O. Borodin, G. D. Smith, T. D. Sewell, D. M. Dattelbaum, and L. L. Stevens, J. Chem. Phys. 131, 224703 (2009).

[7] N. Mathew and T. D. Sewell, Philos. Mag. 95, 424 (2015).

[8] A. A. Rykounov, J. Appl. Phys. 117, 215901 (2015).

[9] D. S. Phillips, R. B. Schwarz, C. B. Skidmore, M. A. Hiskey, and S. F. Son, AIP Conf. Proc. 505, 707 (2000).

[10] D. M. Hoffman and A. T. Fontes, Propellants Explos. Pyrotech. 35, 15 (2010).

[11] B. S. Mallik, I.-F. W. Kuo, L. E. Fried, and J. I. Siepmann, Phys. Chem. Chem. Phys. 14, 4884 (2012).

[12] D. J. Luscher, J. D. Yeager, B. Clausen, S. C. Vogel, A. L. Higginbotham Duque, and D. W. Brown, Crystals 7, 1 (2017).

[13] W. C. Davis, Los Alamos Sci. 2(1), 48 (1981).

[14] M. P. Kroonblawd, N. Mathew, S. Jiang, and T. D. Sewell, Comput. Phys. Commun. 207, 232 (2016).

[15] J. E. Field, Acc. Chem. Res. 25, 489 (1992).

[16] R. Armstrong, H. Ammon, W. Elban, and D. Tsai, Thermochim. Acta 384, 303 (2002).

[17] Q. An, W. A. Goddard, S. V. Zybin, A. Jaramillo-Botero, and T. Zhou, J. Phys. Chem. C 117, 26551 (2013).

[18] N. Mathew and T. D. Sewell, J. Phys. Chem. C 120, 8266 (2016).

[19] R. M. Eason and T. D. Sewell, J. Dyn. Behav. Mater. 1, 423 (2015).

[20] R. A. Austin, N. R. Barton, J. E. Reaugh, and L. E. Fried, J. Appl. Phys. 117, 185902 (2015).

[21] J.-B. Gasnier, F. Willot, H. Trumel, B. Figliuzzi, D. Jeulin, and M. Biessy, Mater. Tech. (Les Ulis, Fr.) 103, 308 (2015).
[22] C. A. Handley, B. D. Lambourn, N. J. Whitworth, H. R. James, and W. J. Belfield, Appl. Phys. Rev. 5, 011303 (2018).

[23] B. W. Olinger and H. H. Cady, https://www.osti.gov/servlets/ purl/7268907.

[24] L. Stevens, N. Velisavljevic, D. Hooks, and D. Dattelbaum, Propellants Explos. Pyrotech. 33, 286 (2008).

[25] N. Mathew, M. P. Kroonblawd, T. Sewell, and D. L. Thompson, Mol. Simul. 44, 613 (2018).

[26] M. R. Taw, J. D. Yeager, D. E. Hooks, T. M. Carvajal, and D. F. Bahr, J. Mater. Res. 32, 2728 (2017).

[27] Y. Long and J. Chen, J. Phys. Chem. C 121, 2778 (2017).

[28] Y. Long and J. Chen, Modell. Simul. Mater. Sci. Eng. 26, 015002 (2018).

[29] Y. Long and J. Chen, J. Appl. Phys. 122, 175105 (2017).

[30] P. Lafourcade, C. Denoual, and J.-B. Maillet, J. Phys. Chem. C 122, 14954 (2018).

[31] C. Denoual, A. M. Caucci, L. Soulard, and Y.-P. Pellegrini, Phys. Rev. Lett. 105, 035703 (2010).

[32] C. Denoual and A. Vattré, J. Mech. Phys. Solids 90, 91 (2016).

[33] A. Vattré and C. Denoual, J. Mech. Phys. Solids 92, 1 (2016).

[34] L. Landau, Phys. Z. Sowjetunion 11, 26 (1937).

[35] V. L. Ginzburg, Il Nuovo Cimento 2, 1234 (1955).

[36] V. Kouznetsova and M. Geers, Mech. Mater. 40, 641 (2008).

[37] V. I. Levitas, Int. J. Solids Struct. 35, 889 (1998).

[38] V. I. Levitas, Int. J. Plasticity 18, 1499 (2002).

[39] V. I. Levitas and M. Javanbakht, J. Mech. Phys. Solids 82, 287 (2015).

[40] B. D. Coleman, Arch. Ration. Mech. Anal. 17, 1 (1964).

[41] L. Soulard, Eur. Phys. J. D 50, 241 (2008).

[42] L. Onsager, J. Am. Chem. Soc. 58, 1486 (1936).

[43] J. Barker and R. Watts, Mol. Phys. 26, 789 (1973).

[44] R. Watts, Mol. Phys. 28, 1069 (1974).

[45] M. P. Kroonblawd and T. D. Sewell, J. Phys. Chem. C 120, 17214 (2016).

[46] L. Verlet, Phys. Rev. 165, 201 (1968).

[47] T. Belytschko, Y. Y. Lu, and L. Gu, Int. J. Numer. Methods Eng. 37, 229 (1994). 